\title{
Particle Adhesion Measurements on Insect Wing Membranes Using Atomic Force Microscopy
}

\author{
Gregory S. Watson, ${ }^{1}$ Bronwen W. Cribb, ${ }^{2}$ and Jolanta A. Watson ${ }^{1}$ \\ ${ }^{1}$ Centre for Biodiscovery and Molecular Development of Therapeutics and School of Pharmacy and Molecular Sciences, \\ James Cook University, Townsville, QLD 4811, Australia \\ ${ }^{2}$ Centre for Microscopy and Microanalysis and School of Biological Sciences, University of Queensland, St. Lucia, \\ QLD 4072, Australia
}

Correspondence should be addressed to Gregory S. Watson, gregory.watson1@jcu.edu.au

Received 21 July 2012; Accepted 8 August 2012

Academic Editors: E. Dague, M. P. Evstigneev, and S. Kasas

Copyright (๑) 2012 Gregory S. Watson et al. This is an open access article distributed under the Creative Commons Attribution License, which permits unrestricted use, distribution, and reproduction in any medium, provided the original work is properly cited.

\begin{abstract}
Many insects have evolved refined self-cleaning membrane structuring to contend with an environment that presents a range of potential contaminates. Contamination has the potential to reduce or interfere with the primary functioning of the wing membrane or affect other wing cuticle properties, (for example, antireflection). Insects will typically encounter a variety of airborne contaminants which include plant matter and soil fragments. Insects with relatively long or large wings may be especially susceptible to fouling due to the high-wing surface area and reduced ability to clean their extremities. In this study we have investigated the adhesion of particles (pollens and hydrophilic silica spheres) to wing membranes of the super/hydrophobic cicada (Thopha sessiliba), butterfly (Eurema hecabe), and the hydrophilic wing of flower wasp (Scolia soror). The adhesional forces with both hydrophobic insects was significantly lower for all particle types than the hydrophilic insect species studied.
\end{abstract}

\section{Introduction}

Many naturally occurring nanostructures, such as the lotus leaf, have functional efficiencies (e.g., self-cleaning) which are similar and/or superior to man-made technologies. One of the most striking natural nanocomposite materials is the insect cuticle [1]. Recently micro- and nanostructures found on insect cuticle have been shown to exhibit a range of impressive and remarkable properties such as superhydrophobicity, ultra-low adhesion, and self-cleaning [2-5]. Distal contamination and wetting of the wings may potentially lead to an increase of the moment of inertia and reduce aerodynamic efficiency [4]. Thus it may be beneficial for long- and/or large-winged insects which are unable to clean their wing extremities to have microstructures which reduce wettability and/or have self-cleaning properties [4]. As well, contamination may impair the functional efficiency of the cuticle.

The atmospheric environment surrounding insects (and indeed humans) contains a complex mixture of natural particulate matter, for example, dust, algae, fungal spores, bacteria, and pollen which can potentially contaminate the insect wing cuticle. Exposure to, and inhalation of, individual or a combination of various air-borne particulates have been found to contribute to various diseases including lung cancer [6]. Pollen grains are generally the most abundant component amongst the floating particles in the air (aeroplankton) surrounding most terrestrial organisms [7]. Other potential airborne contaminants can originate from soils. Soil dust is also a major component of atmospheric particulates. Naturally occurring silica particles composed principally of silicon dioxide $\left(\mathrm{SiO}_{2}\right)$ such as quartz can comprise as much as $90-95 \%$ of the sand and silt fraction of soil [8]. Silica dust has not only been linked to lung disease, but also as a contributor to lung cancer, silicosis, pulmonary tuberculosis, emphysema, and immunologic reaction [9]. The interaction of silica particles as well as pollen grains/fragments with various surfaces is of great interest in terms of distribution, transport, and capture due to the associated health aspects. Insects represent a category of living organisms (especially 
due to their size and features) where enhanced mechanisms for shedding such contaminating particles may be utilized and thus potentially replicated for human applications.

In this study we measure the adhesion of contaminating particles (silica and pollen) on the surfaces of three insect species, that is, cicada (Thopha sessiliba), butterfly (Eurema hecabe), and flower wasp (Scolia soror). The focus of the study is the interaction between particles and the wing structuring.

\section{Experimental}

2.1. Scanning Electron Microscopy. In the case of scanning electron microscope (SEM) imaging, a square of dried wing tissue (approx. $3 \times 5 \mathrm{~mm}^{2}$ ) was excised and mounted on an aluminium pin-type stub with carbon-impregnated double-sided adhesive, then sputter coated with 7-10 nm of platinum, before being imaged using a JEOL 6300 field emission SEM at $8 \mathrm{kV}$.

2.2. Insect Wing Samples. Insect wings were surgically separated by scalpel. The forewings were cut into smaller sections $\left(3 \times 5 \mathrm{~mm}^{2}\right)$ and attached by adhesive tape, or by an epoxy resin, to AFM mounted stubs.

2.3. Instrumentation and Probes. The investigations were carried out with a Thermomicroscope TMX-2000 Explorer/ Discoverer. The instrument is based on detection of tip-tosurface forces through the monitoring of the optical deflection of a laser beam incident on a force-sensing/imposing lever. Scanners were calibrated using atomic scale surfaces and microspheres [10]. The analyses were carried out under air-ambient conditions (temperature of $23-25^{\circ} \mathrm{C}$ and $65-$ $85 \% \mathrm{RH})$.

"Beam-shaped" tipless levers (NT-MDT) were used throughout the work. Typical parameters, as reported by the manufacturer, were the normal force constant $\left(k_{N}\right)$, of the levers was $0.6 \mathrm{Nm}^{-1}$. Particles (silica and pollen) were attached to tipless levers. The procedure has been described previously [11]. The actual cantilever parameters such as the normal force constant were determined using well established methods based on resonance frequency and precalibrated levers [12].

Two relevant particle parameters, namely radius of curvature and nano-scale roughness, were determined quantitatively by SEM and reverse imaging on sharp spikey projections (NT-MDT). The latter technique affords rapid and convenient topographical characterization of a microsphere attached to an AFM probe.

2.4. Force versus Distance (F-d) Analysis. Force versus distance (f-d) analysis was used to obtain adhesion data. The tip/particle is held stationary at an $x-y$ (sample plane) location and is ramped along the $z$-axis, first in the direction of approach and contact with the surface, and then in the reverse direction. F- $d$ curves were acquired at rates of translation in the $z$-direction in the range $5-10 \mu \mathrm{ms}^{-1}$. Each f-d curve consisted of 600 data points. Fifty measurements per particle-substrate size combination were acquired.
A total of 5 particles were attached to cantilevers for each particle type (e.g., five silica beads of $\sim 30 \mu \mathrm{m}$ diameter were used for adhesion measurements each yielding 50 measurements). Only pollen grains (i.e., H. rosa-sinensis sp. "hibiscus" and Euphorbia horrida "snowflake") which exhibited a similar type of orientation upon fixing to a lever were used for adhesion measurements. This was determined using optical microscopy up to a magnification of 600x.

2.5. Adhesion Data. Adhesion was measured under the conditions of the two surfaces coming into contact with no applied loading force, that is, adhesion represented the force of attraction that the particle-cuticle would experience where deformation of structures is minimised and where the main contributing force involved is simply that of the adhesion of the particle to the surface.

\section{Results and Discussion}

3.1. Topographical Characterisation of Insect Wing Cuticle. The insect species and their wing surfaces studied are shown in Figure 1. The micro nanostructuring of the 3 insect species studied are distinctly different. The butterfly (Eurema hecabeFigures 1(a) and 1(b)) topography showed scales with a typical overlaying tile type arrangement as found on many butterfly/moth species [13]. These scales exhibited micron (SEM image in Figure 1(b)) and submicron structuring in the form of longitudinal and lateral elements, as well as globular shaped discrete structures as seen in the SEM image of a single scale in the inset of Figure 1(b). The longitudinal structuring demonstrates a spacing of $\sim 1.8 \mu \mathrm{m}$ with ridges protruding from the surface elements spaced between 1 and $3.5 \mu \mathrm{m}$. The lateral elements are spaced around $1 \mu \mathrm{m}$ apart and the globular bodies are submicron in size (typically less than $400 \mathrm{~nm}$ in any dimension). A number of functional properties have been attributed to scales on butterflies including camouflage display, signalling and possibly thermoregulation control [14-16]. Moth and butterfly scales are typically super/hydrophobic and can detach as an aid for protection against highly adhesive surfaces (e.g., spider webs).

The transparent cicada wing membranes of Thopha sessiliba are covered with a periodic topography. The structures are shown in the topographical SEM image in Figure 1(d). The arrays consist of hexagonally packed spherically capped conical protuberances with a spacing and height of $\sim 200 \mathrm{~nm}$ and radius of curvature of $\sim 25-45 \mathrm{~nm}$ at the apex. Similar features have been found on the wings of a number of other cicada species including Aletta curvicosta, Tamasa tristigma, Macrotristria angularis, Psaltoda Claripennis, and Thopha saccata (e.g., [3]). The features are present on all areas of the dorsal and ventral wing membrane. A previous study has demonstrated the functional effectiveness of similar structures as an antireflective surface which presumably helps to camouflage the insect from predators [3]. The multifunctional nature of the structures appears to be a common feature of the cuticle found on many insect species. 
TABLE 1: Dimensional parameters of all the particles used for adhesion measurements. The values are averaged for 10 particles per particle type.

\begin{tabular}{llcc}
\hline Microsphere supplier & Chemical composition & Actual diameter & Actual surface roughness $\left(1 \times 1 \mu \mathrm{m}^{2}\right.$ area $)$ RMS $(\mathrm{nm})$ \\
\hline Bangs Laboratories & Silica & $4.53 \pm 0.07 \mu \mathrm{m}$ & $3.5 \pm 1.3$ \\
Microspheres-nanospheres Company & Silica & $30.18 \pm 0.16 \mu \mathrm{m}$ & $14.3 \pm 7.2$ \\
\hline
\end{tabular}

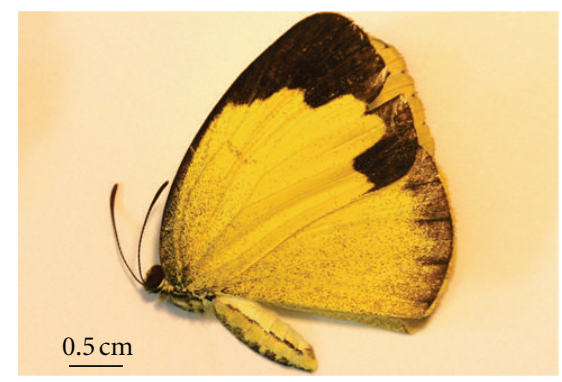

(a)

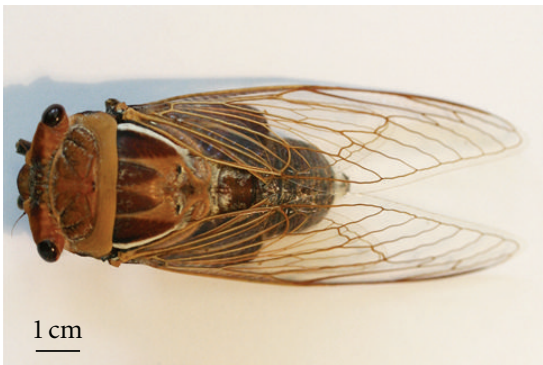

(c)

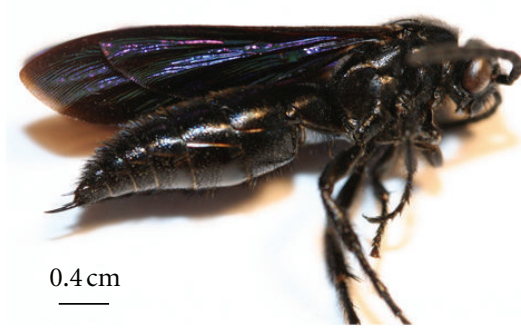

(e)

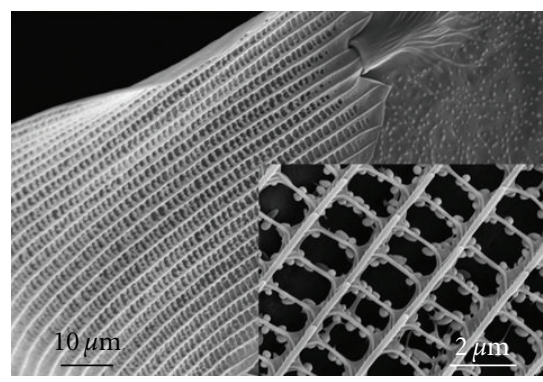

(b)

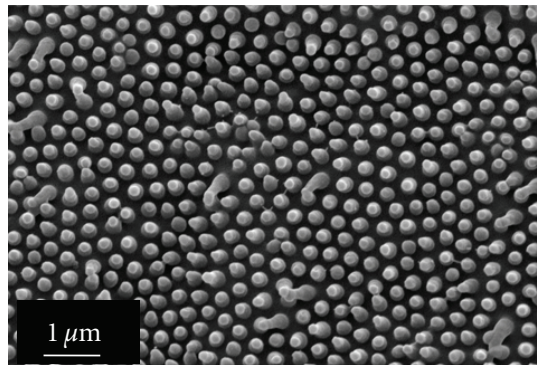

(d)

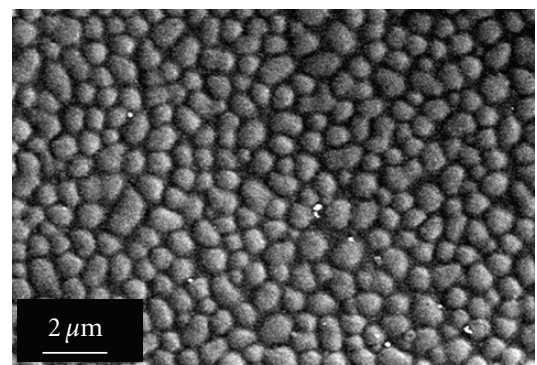

(f)

FIGURE 1: Photographs of the three insect species in this study and corresponding SEM topographical images of the wings. (a) and (b) Butterfly (Eurema hecabe), (c) and (d) cicada (Thopha sessiliba), and (e) and (f) Flower wasp (Scolia soror).

While both the moth and cicada demonstrated superhydrophobicity (static contact angles of $\sim 157^{\circ}$ and $156^{\circ}$, respectively, the flower wasp (Scolia soror) wing was hydrophilic $\left(\mathrm{CA}<90^{\circ}\right)$. The topography was represented by relatively large sized curved projections (bumps), flat (<200 nm in height) and spaced many hundred of nanometres apart (centre-centre distance) (Figure 1(f)). A similar type of insect topography (broad bumps) to this species has been reported in a previous study of micro-/nanostructuring on the termite (Schedorhinotermes sp.) wing membrane $[17,18]$. These studies suggested that the wing membrane topography (array of bumps) may improve flight efficiency by acting as a series of stabilizing elements designed to handle loading forces and also enhance hydrophilic effects.
3.2. Adhesion. The adhesion has been measured on the selected insect species based on the hypothesis that contamination and wettability of wings are related to insect wing size/length. Two-different-sized silica particles have been used to measure adhesion on the insect wing membranes. The dimensional differences (see Table 1) were chosen to mimic contact conditions of particles which could potentially contaminate the structured insect cuticle surfaces. As well, natural particles (2 different pollens, $H$. rosa-sinensis sp. "hibiscus" and Euphorbia horrida "snowflake") were also interacted on the surfaces. Figure 2 also shows SEM images of both the orientation of the particles (contact region facing out of page) used for adhesion measurements and their surface topographies. The pollens were chosen based on 

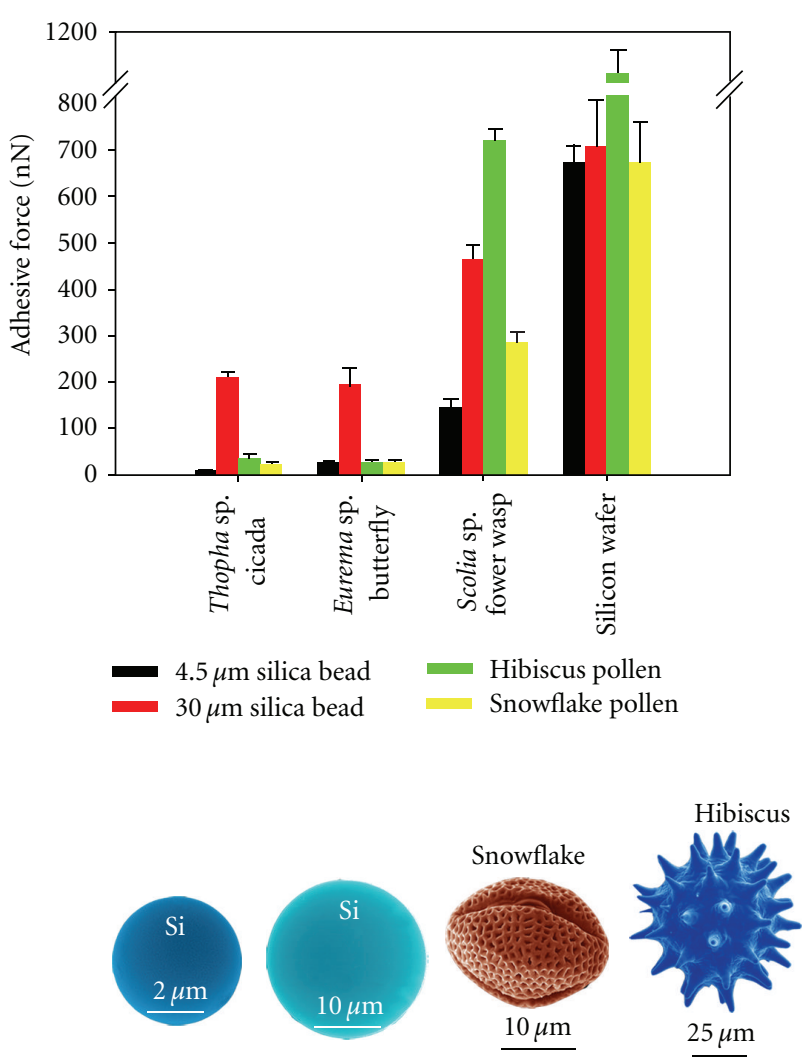

FIGURE 2: Graph displaying the adhesion values of two different silica particle sizes ( 4.5 and $30 \mu \mathrm{m}$ silica beads) with a flat, hydrophilic silicon wafer, and a cicada, butterfly, and flower wasp wing membrane. Falsely coloured SEM images below the graph show the surface topographies of the two silica and two pollen particles used in this study.

the distinct topographies with various levels of roughness. As shown in Figure 2 the spherical $H$. rosa-sinensis sp. hibiscus pollen will present several asperities with the insect cuticles upon contact. The other pollen (Euphorbia horrida snowflake) (Figure 2) demonstrated a homogeneous surface topography/roughness on all sides comprising of small holes and bumps. The outer layer of the pollen grains typically comprises of carboxylic acids cross-linked with saturated and unsaturated aliphatic chains with varying amounts of aromatics resulting in a hydrophobic surface [19].

The adhesion between the various particles and a flat hydrophilic silicon sample with a native oxide layer was used for comparison as it highlights the adhesional differences between the various surfaces. Figure 3 shows representative $f-$ d curves of (a) $4.5 \mu \mathrm{m}$ si bead and (b) snowflake pollen grain interacting with the Thopha sp. cicada and Scolia sp. wasp wing surfaces. For the case where the silica sphere comes into contact with the silicon dioxide surface, meniscus forces at the point of contact between the tip/particle and the surface accounted for the high-adhesive forces.

The adhesion between the silica microsphere and the insect cuticles represents a high-surface energy contaminant particle coming into contact with low-energy hydrophobic and higher energy hydrophilic micro-/nanostructures. This

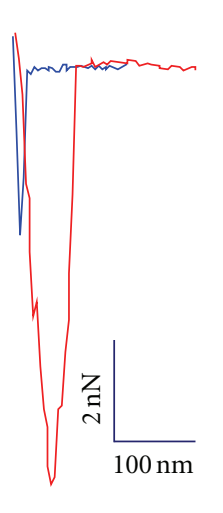

Thopha sp. cicada

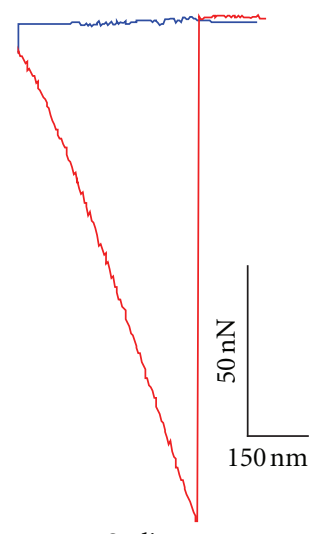

(a) $4.5 \mu \mathrm{m}$ Si bead
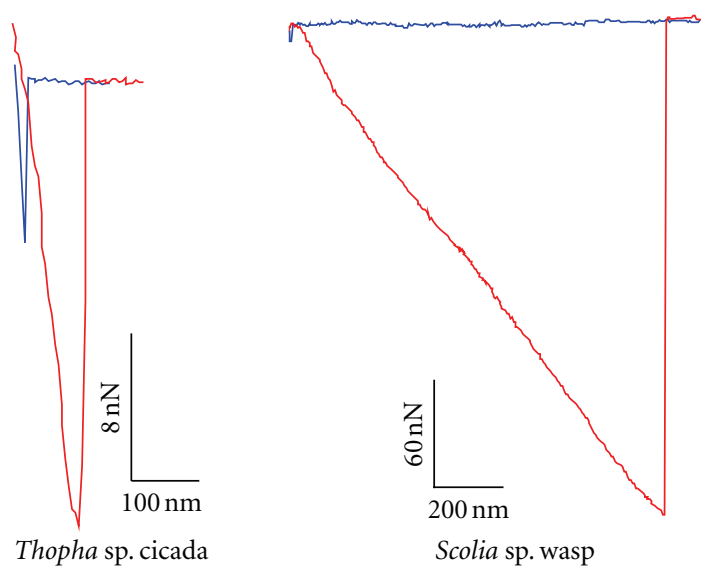

(b) Snowflake pollen

FIGURE 3: Representative f-d curves of (a) $4.5 \mu \mathrm{m}$ Si bead and (b) Snowflake pollen grain interacting with the Thopha sp. Cicada and Scolia sp. wasp wing surfaces. The $\mathrm{f}-\mathrm{d}$ curves show adhesion at a zero loading force.

is highlighted in Figure 2 showing the differences in adhesion between the three insect species. Particle adhesion on the superhydrophobic insect cuticles was much lower in comparison with that for the flat hydrophilic silicon surface and the hydrophilic insect. For comparison, adhesional forces measured on the cicada membrane in an aqueous solution were generally below $10 \mathrm{nN}$ ( $4.5 \mu \mathrm{m}$ silica bead). The higher adhesion values measured in air between the contacting surfaces of the hydrophilic insect membrane/silicon surface and the hydrophilic contaminants reflects the menisci formation from liquid present on the surfaces. As well, the relatively flattened and broadened structures of the hydrophilic insect does not minimise the contact area to the degree of the hydrophobic species.

The larger the particle contact, the higher the adhesion, which reflects the increase in radius of curvature and increased contact points. Thus the real contact area increases along with the meniscus contributions. The "snowflake" pollen grains are of a similar scale to the $30 \mu \mathrm{m}$ silicon beads and thus are useful for comparative purposes. However due to the rougher morphology and more hydrophobic 
nature of the long chain polymers that composes the pollen sporopollenin (outer layer) adhesion between the pollens and the insect cuticles is lower (see Figure 2). The spherically shaped profile with large asperities of the hibiscus exhibited a similar adhesion to the snowflake pollen for interactions with the hydrophobic insect species as shown in Figure 2. The hibiscus was considerably larger in dimensions however only a few of the protuberances will make contact with the insect cuticle and thus contribute to adhesion of the two surfaces.

The highest adhesion between the hydrophilic insect membrane and the pollens was ca. $720 \mathrm{nN}$. Adhesion of the hibiscus pollen was significantly higher on the hydrophilic insect wing and silicon surface. This suggests that liquid bridging forces may play some role in the adhesional contact. The hydrophilic black flower wasp is generally solitary and does not make communal nests. A common feature of this insect is that it is an extremely strong flyer. Thus the insect may have sufficient strength and/or wing flapping frequency to remove contaminants even though the wing membrane is hydrophilic.

\section{Conclusion}

In this study we have evaluated the contact forces of contaminating bodies of different types and sizes by measuring the strength of interaction between particles with selected insect wing cuticle micro-/nanostructuring. An open-framed intricate structuring characteristic of hydrophobic insect species showed minimal adhesion with particles of all sizes. Some of these insects may encounter periods without rainfall and fogging conditions for self-cleaning of wing surfaces. Low adhesion may aid in these circumstances to minimise contamination from foreign bodies and facilitate removal.

In contrast to the hydrophobic species examined the hydrophilic cuticle showed higher adhesion with particles. The unique topographical micro- and nanostructures found on the insect surfaces demonstrate design characteristics and features for tailoring wetting and adhesion to solid body surfaces.

The diversity of the structure topographies demonstrates a range of architectures suitable for optimising surface properties and replication for man-made structures/applications. The hydrophobic insect structures provide a set of wellcharacterised "technologies" which incorporate a range of properties primarily focused on reducing adhesion with solid and water bodies.

\section{Acknowledgment}

Part of this study was funded by a James Cook University Inter University Collaborative Grant (IUCG).

\section{References}

[1] J. F. V. Vincent and U. G. K. Wegst, "Design and mechanical properties of insect cuticle," Arthropod Structure and Development, vol. 33, no. 3, pp. 187-199, 2004.

[2] G. S. Watson, B. W. Cribb, and J. A. Watson, "How micro/nanoarchitecture facilitates anti-wetting: an elegant hierarchical design on the termite wing," ACS Nano, vol. 4, no. 1, pp. 129-136, 2010.

[3] G. S. Watson, S. Myhra, B. W. Cribb, and J. A. Watson, "Putative functions and functional efficiency of ordered cuticular nanoarrays on insect wings," Biophysical Journal, vol. 94, no. 8, pp. 3352-3360, 2008.

[4] T. Wagner, C. Neinhuis, and W. Barthlott, "Wettability and contaminability of insect wings as a function of their surface sculptures," Acta Zoologica, vol. 77, no. 3, pp. 213-225, 1996.

[5] A. R. Parker and C. R. Lawrence, "Water capture by a desert beetle," Nature, vol. 414, no. 6859, pp. 33-34, 2001.

[6] A. M. Knaapen, P. J. A. Borm, C. Albrecht, and R. P. F. Schins, "Inhaled particles and lung cancer. Part A: mechanisms," International Journal of Cancer, vol. 109, no. 6, pp. 799-809, 2004.

[7] H. F. Linskens and M. Cresti, "Pollen-allergy as an ecological phenomenon: a review," Plant Biosystems, vol. 134, no. 3, pp. 341-352, 2000.

[8] J. L. Smith and K. Lee, "Soil as a source of dust and implications for human health," Advances in Agronomy, vol. 80, pp. 1-32, 2003.

[9] M. Ding, F. Chen, X. Shi, B. Yucesoy, B. Mossman, and V. Vallyathan, "Diseases caused by silica: mechanisms of injury and disease development," International Immunopharmacol$o g y$, vol. 2, no. 2-3, pp. 173-182, 2002.

[10] G. S. Watson, B. P. Dinte, J. A. Blach-Watson, and S. Myhra, "Friction measurements using force versus distance friction loops in force microscopy," Applied Surface Science, vol. 235, no. 1-2, pp. 38-42, 2004.

[11] G. S. Watson, J. A. Blach, C. Cahill et al., "Interactions of poly(amino acids) in aqueous solution with charged model surfaces-analysis by colloidal probe," Biosensors and Bioelectronics, vol. 19, no. 11, pp. 1355-1362, 2004.

[12] J. P. Cleveland, S. Manne, D. Bocek, and P. K. Hansma, "A nondestructive method for determining the spring constant of cantilevers for scanning force microscopy," Review of Scientific Instruments, vol. 64, no. 2, pp. 403-405, 1993.

[13] H. Ghiradella, "Structure of butterfly scales: patterning in an insect cuticle," Microscopy Research and Technique, vol. 27, no. 5, pp. 429-438, 1994.

[14] S. N. Gorb, Attachment Devices of Insect Cuticle, Kluwer Academic Publishers, New York, NY, USA, 2001.

[15] P. Vukusic and J. R. Sambles, "Photonic structures in biology," Nature, vol. 424, no. 6950, pp. 852-855, 2003.

[16] Q. Cong, G.-H Chen, Y. Fang, and L.-Q. Ren, "Study on the Super-hydrophobic characteristic of butterfly wing surface," Journal of Bionics Engineering, vol. 1, no. 4, pp. 249-255, 2004.

[17] G. S. Watson and J. A. Watson, "Natural nano-structures on insects - Possible functions of ordered arrays characterized by atomic force microscopy," Applied Surface Science, vol. 235, no. 1-2, pp. 139-144, 2004.

[18] G. S. Watson, B. W. Cribb, and J. A. Watson, "Contrasting micro/nano architecture on termite wings: two divergent strategies for optimising success of colonisation flights," Plos ONE, vol. 6, no. 9, Article ID e24368, 2011.

[19] B. J. R. Thio, J.-H. Lee, and J. C. Meredith, "Characterization of ragweed pollen adhesion to polyamides and polystyrene using atomic force microscopy," Environmental Science and Technology, vol. 43, no. 12, pp. 4308-4313, 2009. 

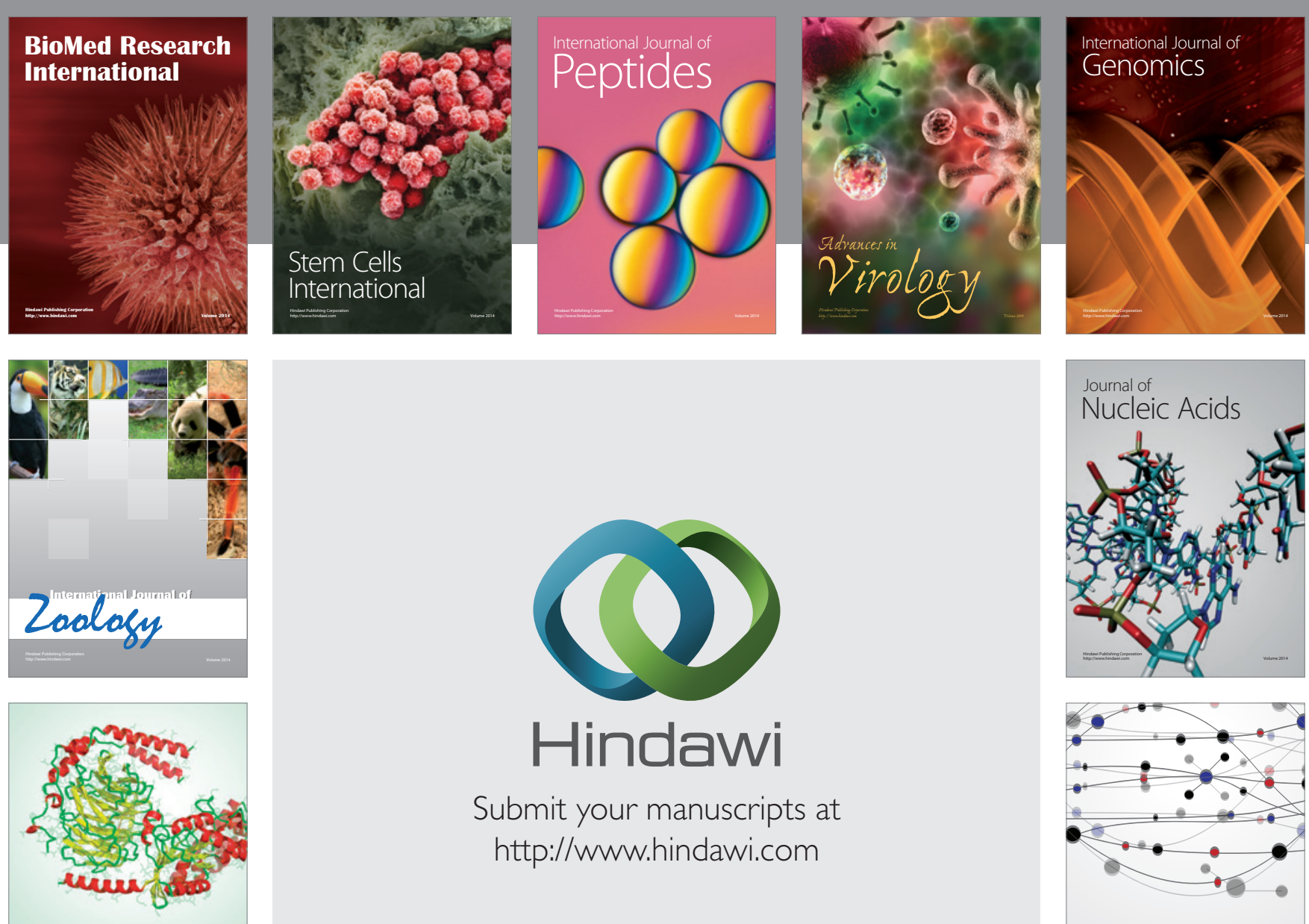

Submit your manuscripts at

http://www.hindawi.com

Signal ${ }^{\text {Jumal }}$ Transduction
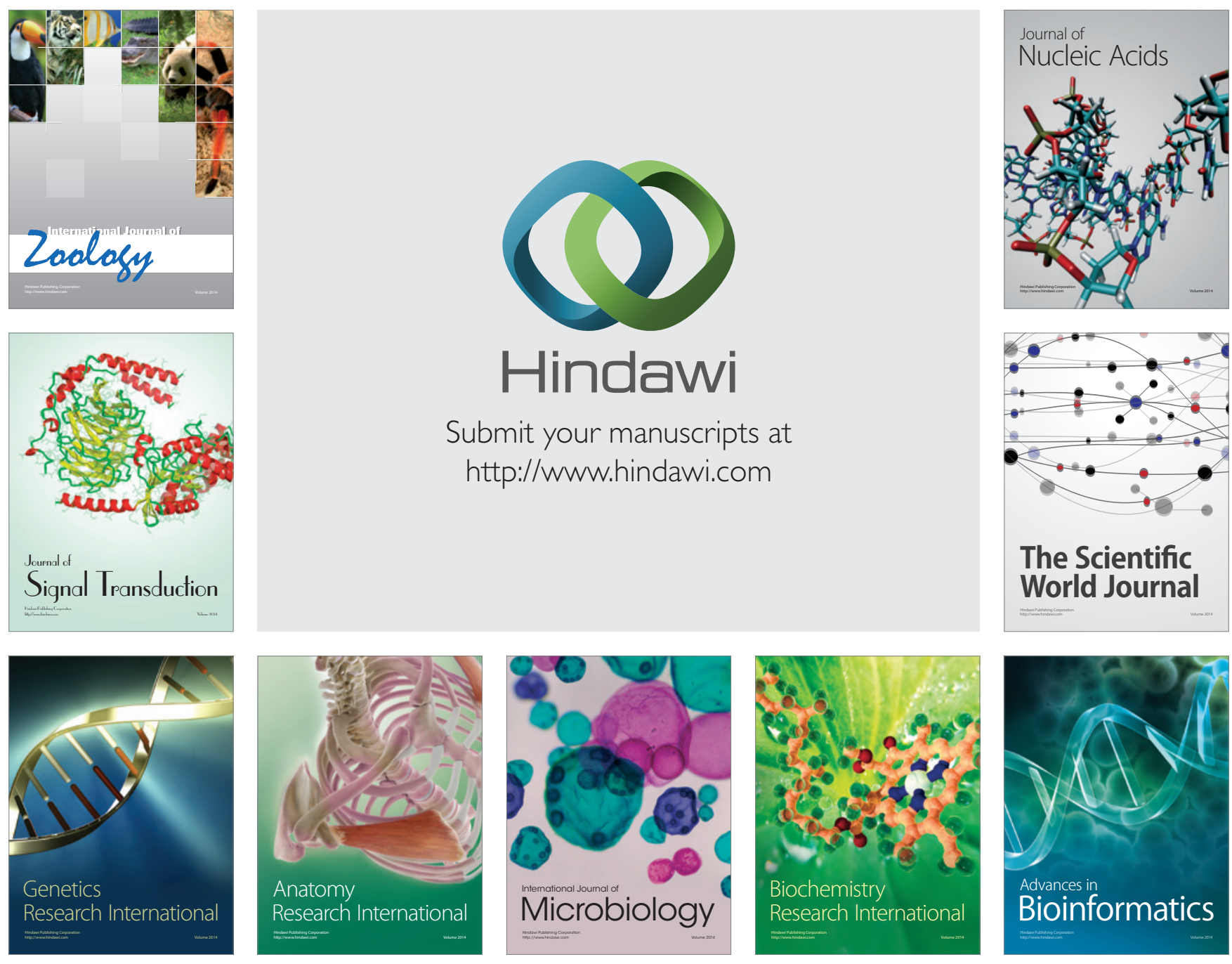

The Scientific World Journal
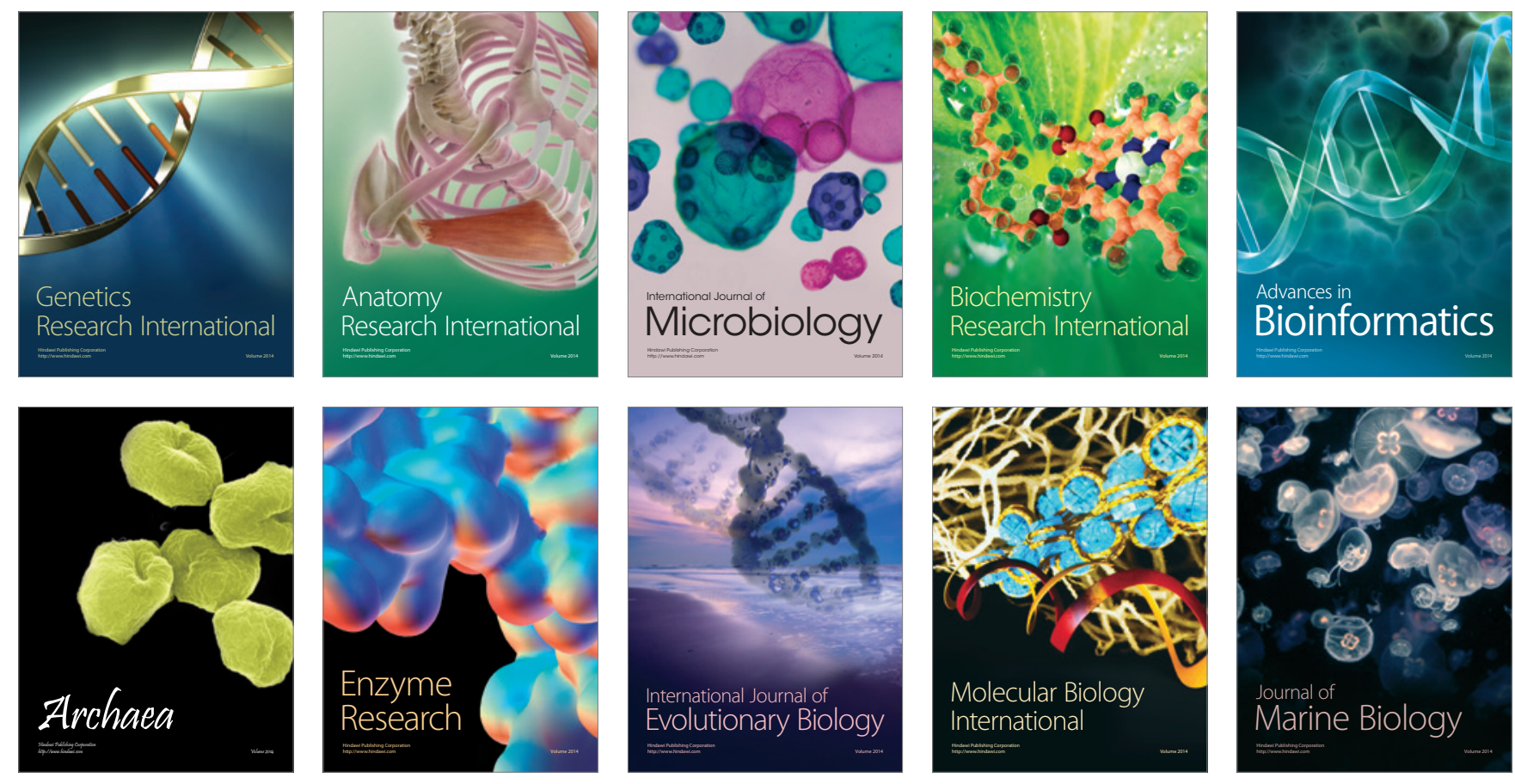